\title{
Les paradoxes de la régénération révolutionnaire
}

Le cas de l'abbé Grégoire

\section{Alyssa Goldstein Sepinwall}

\section{CpenEdition}

Journals

Édition électronique

URL : https://journals.openedition.org/ahrf/182

DOI : 10.4000/ahrf.182

ISSN : 1952-403X

Éditeur :

Armand Colin, Société des études robespierristes

Édition imprimée

Date de publication : 1 septembre 2000

Pagination : 69-90

ISBN : 175 pages

ISSN : 0003-4436

Référence électronique

Alyssa Goldstein Sepinwall, « Les paradoxes de la régénération révolutionnaire », Annales historiques de la Révolution française [En ligne], 321 I juillet-septembre 2000, mis en ligne le 21 février 2006, consulté le 23 avril 2022. URL : http://journals.openedition.org/ahrf/182 ; DOI : https://doi.org/10.4000/ahrf. 182

Ce document a été généré automatiquement le 23 avril 2022.

Tous droits réservés 


\title{
Les paradoxes de la régénération révolutionnaire
}

\author{
Le cas de l'abbé Grégoire ${ }^{1}$
}

Alyssa Goldstein Sepinwall

1 Depuis la panthéonisation d'Henri Grégoire en 1989, l'abbé jadis négligé a bénéficié d'un regain d'intérêt. Cependant, en raison de la grande diversité de ses engagements au cours de sa longue carrière, jusqu'à sa mort en 1831 (il fut prêtre, révolutionnaire, abolitionniste, bibliothécaire, savant, etc.), les historiens ont eu des difficultés à déterminer le sens de sa carrière et le comprendre reste aujourd'hui encore complexe. Des travaux récents ont proposé des évaluations allant des références admiratives de Bernard Plongeron, faisant de Grégoire la "conscience exemplaire" de la France révolutionnaire ${ }^{2}$, aux jugements beaucoup plus critiques d'Annie Kriegel et Samuel Trigano $^{3}$.

2 De telles évaluations contradictoires peuvent-elles être réconciliées? Plutôt que de débattre si l'abbé Grégoire était "bon ou "mauvais, nous avons besoin d'une nouvelle vision d'ensemble de la structure de sa pensée. Nous comprendrons mieux Grégoire - et la Révolution elle-même - en nous concentrant sur les paradoxes incarnés dans son idée de "régénération". La régénération, selon la conception qu'en avait Grégoire, était à la fois libératrice et contraignante pour ceux à qui elle était appliquée. En plaidant pour la régénération de groupes particuliers, et non seulement d'individus, Grégoire a démontré à la fois leur humanité et demandé justice pour eux, mais en même temps il a renforcé l'idée qu'ils étaient dégénérés et inférieurs au reste de la société, et il a ainsi suggéré que ces groupes devraient abandonner leurs particularités culturelles pour pouvoir être régénérés. En plaidant pour la régénération complète de la société, Grégoire aspirait à inaugurer un monde harmonieux de frères mais, dans cette démarche globale, il a cependant été obligé d'opérer des distinctions absolues entre les "régénérables et les "non-régénérables à titre définitif; de même il a été conduit à considérer comme des ennemis de l'État tous ceux qui n'étaient pas d'accord avec ce projet régénérateur. La régénération révolutionnaire pouvait ainsi enfanter des 
mouvements libérateurs pour les individus et les peuples, mais aussi de nouveaux modes de domination et de colonialisme.

Les racines de la régénération sous l'Ancien Régime : Grégoire et les juifs ${ }^{5}$

L'intérêt de Grégoire pour la réforme sociale avait déjà pris naissance au cours des années 1770, lorsqu'il appartenait à une société philanthropique dans l'Est et au moment de la prise de ses premières fonctions ecclésiastiques ${ }^{6}$. Ilessaya alors d'être le modèle d'un "bon curé" éclairé, comme il le nota plus tard dans ses Mémoires: "Je formais le projet de porter aussi loin qu'il est possible la piété éclairée, la pureté des mœurs, et la culture de l'intelligence chez les campagnards, non seulement dans les travaux agricoles, mais en fortifiant leur attachement à ce genre d'occupations ${ }^{7}$. Contrairement aux élites urbaines qui ont voulu "améliorer" les pauvres, Grégoire était lui-même un fils de la campagne; pourtant la haute instruction urbaine qu'il avait reçue comme boursier ${ }^{8}$ l'avait incontestablement éloigné de ses racines rurales et durant sa carrière, il resta comme suspendu entre les mondes urbains et ruraux : il regardait le premier de l'extérieur et critiquait souvent ses valeurs, mais il croyait néanmoins que les gens de la campagne devaient être "améliorés par le savoir des villes ${ }^{9}$.

4 Pendant ces années, Grégoire fit également son entrée dans le monde académique, à Nancy. En 1785, il participa à un concours organisé par la Société royale des sciences et des arts de Metz sur la question: "Est-il des moyens de rendre les juifs plus utiles et plus heureux en France $?^{10}$. Le paradigme qu'il choisit finalement afin de répondre à cette question avait lui-même une histoire complexe. Il s'agissait de la "régénération".

Que signifiait "régénération" au xvirIe siècle ? Jusqu'au milieu du siècle, c'était un mot relativement rare, doté seulement de trois sens: deux théologiques (baptême et résurrection) et un médical utilisé très rarement (réparation des parties blessées du corps). Au milieu du siècle, les philosophes et naturalistes lui ont donné un sens nouveau. $\mathrm{Au}$ moment de la convocation des États généraux, l'occurrence "régénération" est devenue en France un mot à la mode, éloigné de ses connotations baptismales. Par exemple, les pamphlétaires de 1789 célébrèrent Louis XVI comme le "régénérateur de la France", alors que de nombreux textes de cette même période proposaient des méthodes pour régénérer l'État ${ }^{11}$. Selon Antoine de Baecque, "à la veille de la Révolution, c'est l'abbé Grégoire qui définit le plus précisément les usages nouveaux de la régénération avec la version publiée de son essai présenté lors du concours de l'Académie de Metz, l'Essai sur la régénération physique, morale et politique des juifs ${ }^{12}$. Dans cette œuvre, Grégoire a réunifié les définitions théologiques et biologiques anciennes du mot avec ses nouvelles définitions politiques.

6 La première version de la régénération selon Grégoire comprenait déjà le caractère paradoxal que nous verrons tout au long de sa vie et qui lui a permis, à la fois, de défendre et de critiquer les juifs. Dans le climat ouvertement anti-juif de la France de l'Est, et même dans le cercle des amis de Grégoire, dire seulement que les juifs pouvaient être régénérés était déjà une prise de position radicale ${ }^{13}$. Par cette affirmation il était en désaccord avec les autres intellectuels chrétiens (comme Michaëlis, avec lequel l'abbé partageait des amis) qui pensaient que les juifs "sont incapables d'être régénérés, parce qu'ils sont absolument pervers ${ }^{14}$.

7 Pour le prêtre catholique qu'était avant tout Grégoire, bien que les juifs fussent les "plus grands ennemis de [sa] religion ${ }^{15}$, ils étaient des êtres humains. Grégoire avait des amis juifs comme Isaiah Berr-Bing et Simon von Geldern, reconnaissants pour son 
combat sincère pour améliorer leur condition. Il admirait également le célèbre philosophe juif Moses Mendelssohn. Pour Grégoire, les juifs étaient des frères, même si c'étaient des "frères errants. Plutôt que de les "diaboliser", il fallait faire admettre à tous qu'ils faisaient partie de la même famille humaine que les chrétiens.

8 Mais si les juifs faisaient partie de la même famille, comment étaient-ils devenus aussi dégénérés, selon le schéma de pensée de Grégoire à ce moment ? Pour expliquer cette situation, Grégoire blâmait les chrétiens : la dégénération juive était le résultat de la persécution chrétienne et de lois injustes. Il fit appel au gouvernement et aux autres citoyens pour rendre justice aux juifs : "Enfants du même père, dérobez tout prétexte à l'aversion de vos frères... ${ }^{16}$. Il dénonça ceux qui persécutaient les juifs au nom de la religion: "Charité est le cri de l'Évangile, et quand je vois des chrétiens persécuteurs, je suis tenté de croire qu'ils ne l'ont pas lu"17. En plaidant pour la suppression des restrictions civiles arbitraires et des préjugés contre les juifs, Grégoire se fondait en partie sur les arguments déjà développés par d'autres auteurs, comme l'Anglais John Toland, selon qui les juifs devaient être incorporés dans la société sans aucune condition $^{18}$.

9 Mais, contrairement au déiste Toland qui pensait que les problèmes des juifs étaient dus seulement au gouvernement et aux actions des chrétiens ${ }^{19}$, l'abbé Grégoire blâmait également les juifs eux-mêmes, en partie responsables de leur condition. Selon lui, les juifs avaient été rendus inférieurs aux autres hommes non seulement en raison de la persécution des chrétiens et des lois arbitraires, mais aussi à cause des enseignements “ridicules des rabbins et du Talmud. L'emphase de Grégoire sur la "dégénération des juifs renforçait en fait l'idée qu'ils étaient inférieurs par l'abus de pratiques religieuses grotesques : il dénonçait le "culte exclusif des juifs, leur "adhésion obstinée à leurs dogmes et leur "mépris pour les Nations ${ }^{20}$. Il se moquait du Talmud et des croyances des juifs en général: "Si les juifs n'étaient que sauvages [déclara-t-il] on aurait plus de facilité pour les régénérer, il suffirait de semer dans une terre neuve pour espérer une récolte abondante; mais ils ont l'ignorance acquise qui a dépravé leurs facultés intellectuelles. Pour lui, la majorité du peuple juif était "submergée dans un océan de sottes opinions. ${ }^{21}$

10 Grégoire insistait également sur le physique "dégénéré des juifs, qui, selon lui, avait nui à leur caractère moral. S'appuyant sur le travail de Buffon sur la dégénération des espèces, Grégoire suggérait que les juifs avaient subi trop de croisements consanguins. Comparant les humains au monde des animaux, il suggéra que c'était "le croisement dans l'espèce qui abâtardit les races ${ }^{22}$. De plus, il soutint que les juifs devaient abandonner leurs règles religieuses alimentaires, comme les lois kaschers, qui les privaient d'aliments riches en sang ${ }^{23}$. À cette conception biologique de la "dégénération des juifs, Grégoire ajouta les idées physionomiques très répandues de Lavater, qui mettaient en relation le physique et le caractère. Grégoire se révèle avoir été un ardent disciple de Lavater, à qui il avait rendu visite en Suisse : "Le philosophe Lavater, qu'on peut considérer comme législateur quand il sera question de prononcer sur les physionomies, m'a dit avoir observé qu'en général, ils ont le visage blafard, le nez crochu, les yeux enfoncés, le menton proéminent, et les muscles constricteurs de la bouche fortement prononcés. Je me félicite de voir les conséquences morales qu'il en déduit coïncider avec ce que j'ai développé dans le chapitre précédent ${ }^{24}$. Le juif bénéficierait donc de la régénération, suggéra Grégoire, en étant sauvé de lui-même. 
Grâce à l'aide de chrétiens bénévoles, le juif apprendrait à abandonner sa propre ignorance, sa religion et son comportement malsain.

11 Mais la régénération avait un but encore plus urgent: sauver la société des juifs. Décrivant les juifs comme une Nation d'usuriers (bien que la persécution des Gentils les ait réduits à cette condition), Grégoire affirma qu'il était dangereux pour l'État d'avoir trop de juifs vivant en son cœur, et se multipliant rapidement. Bien qu'il pensait qu'en général "une population nombreuse est une source de prospérité pour un État ${ }^{25}$, il usa d'un discours biologique pour invoquer sa crainte d'un temps où "les juifs, devenus trop nombreux, inonderont, infesteront le pays.... ${ }^{26}$. Les titres des chapitres de l'Essai étaient explicites de cette crainte : "Danger de tolérer les juifs tels qu'ils sont à cause de leur population ( chapitre IX); “Danger de tolérer les juifs, tels qu'ils sont, à cause de leur aversion pour les autres peuples, et de leur morale relâchée (chapitre $\mathrm{X}$ ) ou encore "Danger de tolérer les juifs, tels qu'ils sont, à cause de leur commerce et de leurs usures (chapitre XI). Selon Grégoire, beaucoup de membres des classes pauvres de la société, comme ceux parmi lesquels il prêchait, avaient été "ruinés par les juifs ${ }^{27}$. Sans une réforme, pensait Grégoire, les juifs provoqueront un désastre pour la société.

Bien que le principal objectif de Grégoire fut d'assimiler et d'intégrer les juifs dans la Nation, il ne garda pas secret son but le plus important: la conversion. Même si Grégoire dénonçait l'inquisition et autres conversions forcées, il espérait que la tolérance envers les juifs leur ferait adopter volontairement le Christianisme : "À force d'encourager les juifs, insensiblement ils adopteront notre manière de penser et d'agir, nos lois, nos usages et nos mœurs ${ }^{28}$. Il explicitait sa pensée en ces termes: "L'entière liberté religieuse accordée aux juifs sera un grand pas en avant pour les réformer, et j'ose le dire, pour les convertir ${ }^{29}$. Malgré son refus affirmé des conversions forcées, Grégoire n'éprouva pas de gêne à envisager l'obligation pour les juifs à assister à des lectures sur le Christianisme: “Obliger les juifs à s'instruire n'est pas les forcer à se convertir, et je pencherais à croire que les soumettre à l'audition de quelques discours, ce n'est pas contrarier les droits de l'humanité. ${ }^{30}$

Comme nous l'avons vu, le souci de Grégoire à l'égard des juifs ne peut être réduit ni à un simple antisémitisme, ni à leur soutien et à leur défense inconditionnelle. Le but de la "régénération était d'aider un groupe humain à accéder à un état meilleur, en utilisant l'arme des Lumières et du Christianisme, ce qui confirmait clairement que le groupe en question était "dégénéré. Ainsi, afin d'être vraiment régénérés, les juifs devront commencer par changer leurs mœurs et ce sera seulement après cette mutation qu'ils pourront s'élever au niveau du reste de la société.

Avec la Révolution : régénérer la Nation, régénérer ses composantes

14 Avec la chute de la Bastille, la régénération prenait un sens nouveau et devenait une urgente nécessité. Grégoire devint rapidement un des députés les plus influents grâce à sa forte éloquence et son enthousiasme de jeunesse ${ }^{31}$. Ses discrets projets prérévolutionnaires s'étendirent, dans l'agitation générale de cette période où tout semblait pouvoir être réformé, en un vaste projet de reconstruction de la France entière. Grégoire joua un rôle clé dans les discussions de l'Assemblée et il commença à se lier à quelques-uns des députés les plus radicaux. Cependant, ses espoirs d'une synthèse harmonieuse entre les Lumières et la vision chrétienne du monde l'amenèrent parfois à prendre des positions différentes de celles défendues par ses nouveaux amis ${ }^{32}$. $\mathrm{Au}$ cours d'un sermon, en 1789 , Grégoire évoqua l'utopie qu'il espérait voir surgir de la Révolution en cours: "Dans un avenir prochain, je vois tous les canaux de l'agriculture 
et du commerce s'ouvrir pour y faire circuler l'abondance... tous les arts, dégagés d'entraves, déploient leur énergie... le Christianisme recouvre sa splendeur; l'éducation régénérée répare les outrages faits à la majesté des autels, et le xixe siècle s'ouvre sous les auspices du bonheur ${ }^{33}$. Tout en énonçant cette nouvelle perspective pour la société française entière, Grégoire se concentrait, pendant cette période, sur des groupes particuliers qui avaient besoin d'une régénération particulière.

Bien qu'il ait soulevé très tôt le problème des juifs, Grégoire garda le silence à l'Assemblée lors de la plupart des débats les concernant ${ }^{34}$. En revanche, il appliqua une grande part de son énergie à la régénération du peuple des campagnes. Il se fit un des plus ardents propagateurs des efforts destinés à répandre les techniques agricoles nouvelles dans les campagnes, et il essaya d'aider les paysans à conserver leurs forêts communales ${ }^{35}$.

16 Au moment même où Grégoire luttait pour alléger le fardeau des gens de la campagne, il suggérait qu'ils avaient également besoin d'une régénération spéciale, surtout s'ils parlaient le patois. En 1788 déjà, Grégoire avait indiqué que "l'anéantissement des patois importe à l'expansion des lumières, à la connaissance épurée de la religion, à l'exécution facile des lois, au bonheur national, et à la tranquillité politique ${ }^{36}$. En 1790, il envoya des questionnaires dans toute la France, et demanda comment les patois pouvaient être extirpés. Il était particulièrement inquiet de l'influence que pouvaient avoir les patois sur les mœurs, la religiosité, et les préjugés des gens ${ }^{37}$. Bien avant que la plupart des autres dirigeants de la Révolution ne considèrent la lutte contre les patois comme un thème essentiel de l'action politique à venir, Grégoire insistait pour souligner que l'ignorance de la langue française chez les paysans pourrait faciliter une Contre-Révolution ${ }^{38}$. Ainsi, alors même que Grégoire souhaitait préserver les mœurs rurales idéalisées des gens de la campagne, ses suggestions pour l'amélioration de leur langage impliquaient qu'ils devaient imiter leurs frères des villes et abandonner leurs traditions culturelles et linguistiques particulières.

Ce fut à cette même période que Grégoire devint le défenseur intrépide des gens de couleur dans les colonies françaises. Après avoir condamné, à l'automne de 1789, certains philanthropes plus soucieux des Noirs à "deux milles lieues d'existence ${ }^{39}$ que des juifs vivant sur le territoire français, il changea d'avis après avoir rencontré les députés de couleur venus en France protester contre leur exclusion des assemblées coloniales. Il devint dès lors un violent dénonciateur de "l'aristocratie de la couleur ${ }^{40}$; position parfaitement symétrique à son opposition antérieure aux lois arbitraires qui avaient expulsé les juifs des principales activités de l'économie et de la société. Il dénonça ainsi les lois propres aux colonies, qui interdisaient aux gens de couleur de pouvoir manger à la même table que celle des Blancs ${ }^{41}$. Malgré les accusations ultérieures selon lesquelles il aurait provoqué les révoltes des esclaves et des libres de couleur à Saint-Domingue en 1790-1791, Grégoire continua sa lutte pour l'égalité des droits des hommes de toutes les couleurs sous l'Empire, la Restauration et jusqu'à l'extrême fin de sa vie.

18 Ici aussi, cependant, l'idée de régénération formulée par Grégoire impliquait la nécessité d'une homogénéisation et d'une imitation. Quel qu'ait été à ses yeux le potentiel des colonies pour "l'éducation régénérée [et] des mœurs purifiées" ${ }^{42}$, cela demeurait seulement une possibilité future, et non une réalité immédiate. Pour réaliser ce potentiel, la première étape devait d'abord consister en une œuvre de "civilisation" des gens de couleur des colonies, ainsi que de leurs frères d'Afrique. Comme Grégoire le 
nota en 1796, les hommes de couleur avaient besoin d'une "révolution morale afin qu'ils puissent "sentir leur dignité d'hommes libres".

Si les hommes libres de couleur pouvaient être immédiatement régénérés, Grégoire pensait cependant que les esclaves étaient tellement dégénérés qu'ils ne pouvaient pas encore participer à la citoyenneté. Alors même qu'il invoquait l'universalisme de la Déclaration des droits en faveur des mulâtres, Grégoire affirmait que les esclaves noirs ne pouvaient pas encore accéder pleinement à ces droits. Utilisant le langage des devoirs, reflétant une de ses différences avec les autres radicaux, Grégoire affirma en mai 1791 que leur cause "n'a rien de commun avec celle des mulâtres... il ne faut rien brusquer, et... donner tous les droits politiques à des hommes qui n'en connaissent pas tous les devoirs, ce serait peut-être mettre une épée entre les mains d'un furieux... ${ }^{44}$. En fait, l'un des arguments de Grégoire en faveur des libres de couleur était qu'ils pouvaient aider les Blancs à "contenir les esclaves ${ }^{45}$ et à contrôler le marronnage ${ }^{46}$. Comme d'autres Amis des Noirs, Grégoire n'était pas favorable à une émancipation immédiate, car il pensait que les esclaves avaient besoin d'une période de régénération spécialement longue avant qu'ils ne puissent être au niveau des autres. À cette fin, Grégoire encourageait les libres de couleur à étendre le Christianisme parmi leurs propres esclaves "en les amenant progressivement à la libertée $e^{47}$.

Quelques contemporains de Grégoire reconnurent le paradoxe d'une régénération ostensiblement universelle mais dont l'application à des groupes particuliers supposait des "mesures spécifiques. Les juifs "acculturés" de Bordeaux, qui ne voulaient pas se distinguer de leurs voisins, demandèrent expressément à Grégoire de cesser de préconiser des lois particulières pour les juifs; placer les juifs en dehors de la régénération générale de la France serait "une injustice aussi gratuite qu'elle serait cruelle $^{48}$. Pareillement, en 1790 , les chefs de la Commune de Paris rappelèrent à l'Assemblée que "proposer de régénérer les juifs par des règlements particuliers, n'est donc qu'un effort du préjugé pour enchaîner votre sagesse et votre justice... les juifs, comme les chrétiens, doivent être régénérés par la Constitution [seule]. ${ }^{49}$

Consolider la Révolution : régénérer la Nation, créer des ennemis

21 Pendant les premières années de la Révolution, Grégoire avait pensé qu'éliminer les mauvaises institutions, y compris des mesures de restrictions légales arbitraires contre certains groupes, serait suffisant pour déclencher la régénération de la Nation entière. De même il avait cru qu'avec la nouvelle Constitution la société entière serait libérée tout à coup de la corruption de l'Ancien Régime ${ }^{50}$ et que toutes les distinctions arbitraires fondées sur le privilège ou le préjugé disparaîtraient.

Lorsque la Constitution fut achevée et les lois injustes supprimées, Grégoire consacra son énergie à la régénération par la diffusion de cette Constitution dans le corps social entier, sans limiter son attention à des groupes particuliers. Lors de son séjour à Blois comme évêque constitutionnel, il s'appliqua à réaliser la synthèse chrétienne révolutionnaire qui demeurait au cœur de sa vision de la régénération ${ }^{51}$. La régénération promettait la renaissance de la Nation entière et, à terme, de toute l'humanité. Mais à mesure que le temps passait, la promesse de régénération s'atténuait et ses aspects paradoxaux devenaient de plus en plus sensibles par des manifestations nouvelles. Quand il partit dans les départements, d'abord à Blois en tant qu'évêque constitutionnel, puis à Chambéry et à Nice comme commissaire de la Convention, Grégoire fut consterné de découvrir que certaines personnes refusaient d'adopter le nouvel ordre. Alors que se répandaient des rumeurs de guerre et que la 
Révolution semblait menacée, rester neutre envers le nouveau régime n'était plus une attitude envisageable à ses yeux.

Bientôt, la régénération devint - pour Grégoire ainsi que pour d'autres révolutionnaires - un critère de distinction parfaitement efficace : c'était un moyen de reconnaître ceux qui étaient les hommes nouveaux révolutionnaires de ceux qui restaient des hommes de l'Ancien Régime. Grégoire fut un des premiers à transformer les idéaux utopiques de la régénération en offensives dirigées contre les ennemis de la Révolution. Toute personne non disposée à accepter la régénération révolutionnaire devait être éliminée ou la Révolution serait en péril. Les actions de Grégoire ont coïncidé de plus en plus avec le deuxième modèle de régénération décrit par Mona Ozouf : non plus celui de ceux qui voyaient la régénération comme un miracle spontané accompli en 1789, mais celui de ceux qui la concevaient comme un combat interminable pour détruire les débris corrupteurs du passé monarchique ${ }^{52}$. Le vocabulaire de Grégoire s'emplissait alors de mots comme "anéantir, "purger, "effacer et "épurer".

L'attitude de Grégoire par rapport aux prêtres réfractaires montre bien la façon dont l'idéal utopique de la régénération pouvait provoquer une lutte acharnée. Ayant initialement été hésitant lui-même à soutenir la Constitution civile du Clergé (il craignait que son rejet par le Pape ne produisît un schisme), Grégoire fut tout d'abord tolérant envers les réfractaires. En janvier 1791, il avait déclaré devant l'Assemblée que les réfractaires avaient de bonnes intentions: "Parmi les ecclésiastiques... qui se trouvent dans cette Assemblée, les uns ont prêté leur serment, les autres s'y sont refusés; de part et d'autre nous devons supposer des motifs respectables ${ }^{53}$. Il pensait qu'il pouvait vaincre ceux qui doutaient en les persuadant que "Nous déclarons ne rien apercevoir dans la constitution qui puisse blesser les vérités saintes que nous devons croire et enseigner. Ce serait injurier, calomnier l'Assemblée nationale, que de lui supposer le projet de mettre la main à l'encensoir ${ }^{54}$.

25 Mais, quant il devint évident que beaucoup dans l'Église s'opposeraient à la régénération révolutionnaire, Grégoire ne put plus accepter de considérer l'opposition des prêtres réfractaires comme sincère. En mars 1791, dans une lettre pastorale de Blois, Grégoire attaqua les réfractaires comme "ennemis de la patrie ${ }^{55}$. Invoquant le spectre de complots contre-révolutionnaires, il commença par déclarer que la religion n'était pour eux qu'un prétexte : “La religion est un voile sacré dont les pervers abusent pour couvrir leurs complots... la seule hérésie qu'ils trouvent dans la constitution, c'est l'égalités6.

Grégoire indiquait également, dans ce même texte, que les aristocrates ne pouvaient pas être régénérés et avaient perdu leurs droits de membres de la Nation. Dès le mois d'août 1790, Grégoire avait affirmé que "l'horreur que nous inspire la conduite des émigrants nous absout de les avoir pour compatriotes; et plût à Dieu que la France entière pût vomir de son sein tous les ennemis qu'elle y recèle ${ }^{57}$. En 1793, Grégoire suggérait que les aristocrates en tant que catégorie ne pouvaient être régénérés, quoi qu'ils fassent. "L'aristocratie est une maladie incurable, déclara-t-il ${ }^{58}$.

27 Dans le même sens, Grégoire sentait qu'il n'était pas suffisant d'abolir la monarchie en France seule : toutes les monarchies devaient être rayées de la terre. Le langage de plus en plus violent de Grégoire venait à la fois d'une conscience aiguë de la nécessité de voir les valeurs de la Révolution française adoptées par les autres peuples et de la poursuite de la guerre. À la suite du 10 août 1792, il proclama : "Il s'agit d'exterminer le despotisme, d'anéantir son orgueil stupide, de purger la terre, de broyer ces monstres 
qui se disputent les lambeaux des hommes, de révéler à tous les peuples leurs droits imprescriptibles, et d'affranchir l'espèce humaine ${ }^{59}$. Après avoir proposé l'abolition de la monarchie ("toutes les dynasties n'ont jamais été que des races dévorantes qui ne vivaient que du sang des peuples... il faut détruire ce mot de roi ${ }^{60}$, il proclamait que tous les hommes, dans le monde entier, devraient faire le même geste. Grégoire deviendra par la suite un des conventionnels les plus enclins à acclamer les délégations étrangères venues apporter leur soutien à la Convention ${ }^{61}$. À ses yeux l'existence même de la jeune République française serait en danger si les autres Nations restaient dans la béatitude royale.

Tout en essayant d'atteindre le vaste objectif de la régénération générale, Grégoire commençait à restreindre le cercle des "vrais patriotes". Au début de la guerre, il en appela aux citoyens de Blois de ne pas "s'enfermer lâchement dans leurs maisons à l'intérieur du pays, mais d'aller aux frontières et de défendre la France avec leur vie : "Vous avez juré de vivre libres ou mourir: le moment est arrivé de remplir vos serments..." ${ }^{62}$. Pendant sa mission dans le nouveau département des Alpes-Maritimes, Grégoire déclara - exactement comme d'autres jacobins le faisaient à la Convention que "les demi-patriotes sont souvent aussi dangereux que les contre-révolutionnaires ${ }^{63}$. Avec le temps, il décida que les demi-patriotes étaient "plus dangereux encore, sous le masque qui les couvre, que les aristocrates déclarés ${ }^{64}$. Il fit des distinctions entre les vrais patriotes et les autres, et indiqua que les premiers devaient écraser les derniers : "Il faut que les vrais amis de la République se concertent pour écraser tous ceux qui tentent par un moyen quelconque d'altérer la pureté des principes. ${ }^{65}$

Dans la quête de Grégoire pour la régénération, il n'avait pourtant jamais souhaité oublier complètement le passé. Après tout, en tant que fervent chrétien, une grande part de sa pensée reposait sur les enseignements de Jésus et les récits bibliques. Contrairement à d'autres radicaux, Grégoire voulait prendre le meilleur du passé (en particulier les principes chrétiens et républicains classiques) et le combiner avec le présent.

30 Mais lorsque Grégoire retourna à la Convention après sa mission, il commença à comprendre jusqu'à quel point beaucoup de ses alliés de longue date avaient conçu la régénération comme une rupture complète avec l'histoire. Lorsque le Comité d'instruction publique commença à recevoir des rapports venus de départements et de villes où des livres rares étaient brûlés, l'intellectuel qu'il était fut horrifié. Il ne concevait pas que beaucoup de ses amis, membres du Comité, puissent être satisfaits de voir les livres être ainsi détruits pour la seule réutilisation du papier ${ }^{66}$. Bien qu'il ait autrefois encouragé la destruction de certains monuments monarchiques, la profanation des églises et des livres rares le choquait. À ce moment l'évêque constitutionnel qu'il était encore décida que les appels à la régénération étaient devenus trop radicaux et étaient détournés de leur objectif initial. Il commença à dénoncer cette destruction totale du passé et devint le principal, sinon le premier, critique du "vandalisme"67.

31 En brumaire an II, lorsque d'autres conventionnels déclarèrent clairement que la régénération impliquerait désormais une répudiation totale du Christianisme, de nouveaux dilemmes apparurent pour Grégoire de manière particulièrement douloureuse. Forcé de choisir entre le Christianisme et la République, qu'il voyait comme indissociables, Grégoire refusa ; il déclara que son Christianisme était partie intégrante de son être et qu'il ne pouvait l'abandonner. Dans les années qui suivirent 
immédiatement le 9 Thermidor an II, Grégoire employa une grande part de son énergie à revitaliser l'Église constitutionnelle. Il décida que la France pouvait être régénérée seulement sur la base de cette institution, incarnant un Christianisme républicain modéré68.

Après la Révolution : globaliser la régénération

Avec la fin de la Révolution et la signature du Concordat entre Napoléon et le Pape, Grégoire se trouva refoulé aux marges du pouvoir. Désormais il était conscient qu'il ne pourrait plus influer sur le cours de la régénération nationale : la Révolution et l'Église constitutionnelle n'existaient plus. Mais bien que l'énergie frénétique des années révolutionnaires se soit éteinte, Grégoire n'abandonna pas ses espoirs de régénérer le monde, alors que d'autres révolutionnaires - comme Roederer et Sieyès - qui partagaient, eux aussi, des rêves utopiques de régénération perdirent toute illusion et se rallièrent à Napoléon. Toutefois Grégoire, peut-être parce qu'être un prêtre lui avait appris que la patience était nécessaire pour convertir des âmes, resta ardemment attaché au républicanisme. Se regardant lui-même comme le seul qui n'avait pas changé, Grégoire entreprit de préserver et de propager l'héritage révolutionnaire.

Il chercha ainsi d'autres voies pour accomplir une régénération républicaine, mais stable et chrétienne. Une partie de son temps fut employée à l'écriture de l'histoire, particulièrement celle des idées chrétiennes et républicaines. Puisque la régénération devait se fonder sur le passé et non sur sa destruction, il était essentiel que les gens puissent le comprendre ${ }^{69}$.

34 L'autre versant important de son engagement et de son énergie était orienté vers l'étranger et tout particulièrement vers la périphérie de l'Europe et du Nouveau Monde. Alors qu'il était autrefois préoccupé par les groupes opprimés dans l'Empire français, il faisait maintenant campagne pour les gens opprimés (qu'il appelait les malheureux) partout, de l'Irlande à l'Inde et à l'Afrique. Le commerce des esclaves devint une de ses préoccupations principales. Il se concerta avec des hommes et des femmes dans le monde entier pour abolir la traite des Noirs. Il publia De la littérature des nègres, un livre largement diffusé, soulignant les accomplissements culturels atteints par des personnes de descendance africaine. La censure ayant empêché Grégoire de condamner explicitement le rétablissement par Napoléon de l'esclavage aux Antilles, Grégoire chercha à détruire indirectement les fondations de cet esclavage rétabli, en démontrant l'humanité des Noirs.

35 En plus des malheureux, Grégoire se concentra sur les républiques émergentes dans le monde entier. Avec l'échec de la République française, ces nouvelles républiques représentaient alors à ses yeux le futur du monde et il était déterminé à les empêcher de suivre l'exemple français : il voulait qu'elles soient à la fois égalitaires et inspirées de la morale chrétienne. En Europe, il se concentra particulièrement sur les républicains grecs $^{70}$. Grégoire s'intéressa également au Nouveau Monde, et aux jeunes États-Unis. Bien qu'il soit attiré par les idéaux politiques de cette nouvelle république, il était déçu que les Américains aient permis l'institutionnalisation de l'esclavage dans leur constitution. Il communiqua sa consternation aux premiers intellectuels américains comme Thomas Jefferson et essaya d'aider les forces abolitionnistes dans ce pays ${ }^{71}$. Il resta également vivement intéressé par le progrès du catholicisme et l'intégration des juifs aux États-Unis ${ }^{72}$.

36 Ici encore, ses actions pouvaient s'avérer libératrices pour les peuples à régénérer. L'emphase de Grégoire sur l'unité de l'espèce humaine et l'humanité des peuples non 
occidentaux contrait les idéologies racistes des esclavagistes et de ceux qui les assimilaient à des animaux. Dans un climat intellectuel où les naturalistes commençaient à affirmer que la "race" blanche était biologiquement et irrévocablement supérieure à toutes les autres, Grégoire était calomnié pour son obstination à continuer à affirmer que les différences entre les personnes étaient le résultat de facteurs culturels et de circonstances historiques. Grâce à la puissante logique des attaques de Grégoire contre le racisme, des livres tels que De la littérature des nègres avaient valu à l'abbé une admiration soutenue des peuples du monde entier, dans la longue durée. Par exemple on vit, en 1950, Ho Chi Minh acclamer Grégoire comme "l'apôtre de la liberté des peuples ${ }^{73}$ et Aimé Césaire le vanter comme "le premier réfutateur scientifique du racisme et "le premier militant de l'anti-colonialisme ${ }^{74}$. Une large variété de groupes - du Sénégal à l'Amérique - s'inspira des luttes de Grégoire pour les groupes opprimés du monde entier et de son soutien pour les Nations nouvellement indépendantes.

Cependant, les qualités émancipatrices des idéaux de Grégoire ne doivent pas en masquer les limites. Le soutien de Grégoire envers les malheureux était fondé sur l'idée qu'ils devaient "rejoindre" l'Église catholique ${ }^{75}$. La déception post-révolutionnaire de Grégoire par rapport aux juifs français est révélatrice. Il était frustré qu'ils aient obtenu des droits sans pour autant se régénérer ${ }^{76}$. Il condamnait leur attachement au Talmud qu'il voyait comme une collection "qui rappelle ce qu'Horace a dit de quelques perles égarées dans le fumier d'Eunius - et il dénonçait fortement le "despotisme des rabbins et des dirigeants des communautés dans deux articles qu'il publia en 1806 et 1807 . Il encourageait les juifs à "secouer courageusement le joug du rabbinisme $e^{77}$, position pour laquelle il fut applaudi par des amis comme son vieil ami Frédéric-Rodolphe Saltzmann qui lui dit: "Vous ne flattez pas les juifs de notre pays. Mais ce que vous en dites est très $\mathrm{vrai}^{78}$. Pareillement, Hannah Adams, une missionnaire américaine auprès des juifs, emprunta beaucoup aux écrits de Grégoire et fut même encouragée par une de ses lettres l'assurant que: "From time to time the Jews of both sexes have been converted at Metz ${ }^{79}$. Malgré sa déception quant à la rapidité de régénération des juifs français, Grégoire continua d'espérer que des solutions pourraient être trouvées pour "leur donner un caractère homogène de civilisation avec les autres citoyens ${ }^{80}$. Il garda espoir en ce que "les juifs reviendront à celui que leurs aïeux ont percés1.

Ces éléments restrictifs de l'attitude de Grégoire ne passèrent pas inaperçus auprès des juifs contemporains. Zalkind Hourwitz s'était hérissé en 1788 lorsque Grégoire avait suggéré que les juifs avaient besoin de correction ${ }^{82}$ de même le très influent juif BerrIsaac-Berr fut affligé par le nouveau coup d'éclat de Grégoire. Berr, qui avait travaillé avec Grégoire dans la lutte pour la citoyenneté des juifs pendant la Révolution, rappela "hautement la gratitude et la profonde estime dont [il était] pénétré pour [lui]. Cependant il put à peine contenir sa colère envers la dernière critique de Grégoire sur le Talmud. Il affirma que d'attaquer les livres sacrés d'une religion, "c'est d'attaquer la religion même à laquelle ils appartiennent... il serait extrêmement aisé de jeter du ridicule sur la plupart des livres que les diverses sectes du christianisme admettent comme sacrés. Mais, il demanda, "Que suit-il de là, M. le Sénateur?. Berr concluait que les plans de Grégoire transformeraient les juifs en non-juifs pour en dénoncer les dangers: "des invitations à renoncer totalement au joug du rabbinisme leur [aux juifs] soient répétées par des hommes respectables tels que vous, Monsieur, bientôt la confusion s'en suivra; et ces individus, oubliant alors la religion de leurs ancêtres, ne seront ni juifs, ni catholiques, ni protestants, et ne feront qu'augmenter le nombre des 
athées, ou des hommes sans principes moraux certains... Serait-ce là un avantage pour le gouvernement ?33. Malgré ces doutes exprimés envers Grégoire, les juifs contemporains n'avaient pas beaucoup d'autres alliés. Reconnaissants envers Grégoire pour sa condamnation de la violence exercée à leur encontre, les juifs essayèrent de ne pas tenir compte des aspects négatifs de sa conception de la régénération en retenant surtout l'affirmation qu'ils pouvaient être considérés comme les égaux des autres citoyens. Ils brandirent ainsi le slogan de la régénération comme un moyen de surmonter l'héritage d'une discrimination passée ${ }^{84}$.

Les paradoxes de la régénération se révèlent également bien dans la relation de Grégoire avec Haïti. Se fondant sur les contacts qu'il avait entretenus de longue date avec Saint-Domingue, Grégoire se présenta comme un père, vieux et sage, pour Haïti. Il imagina la République d'Haïti régénérée comme un "phare" pour le monde, un brillant exemple du potentiel républicain. En correspondance avec des hommes et femmes de couleur fortunés, particulièrement dans la République du Sud dirigée par les Métis, Grégoire adjura les Haïtiens de ne pas commettre les mêmes erreurs que la France avait faites, et élabora pour eux une voie à suivre ${ }^{85}$.

Dans sa correspondance privée et dans ses œuvres imprimées, Grégoire était même plus avancé que la plupart des autres abolitionnistes à bien des égards, y compris par son soutien à la souveraineté haïtienne, son respect envers ses chefs politiques, et son refus de tous les préjugés de peau. Plutôt que de se différencier des infortunés qu'il essayait de défendre, il déclara sa parenté avec eux : "J'ai pour ainsi dire identifié mon existence avec celle des Haïtiens ${ }^{86}$ Grégoire critiqua la dépravation de la société européenne mondaine, se demandant, comme Raynal et d'autres l'avaient fait avant lui, si l'immoralité de certains des aspects de la civilisation européenne signifiait réellement un progrès sur les mœurs "sauvages ${ }^{87}$. Pour lui, Haïti pouvait être un modèle pour le reste du monde : "Haïti libre est un phare élevé sur les Antilles vers lequel les esclaves et leurs maîtres, les opprimés et les oppresseurs tournent leurs regards ${ }^{88}$. Grâce à son inébranlable soutien, Grégoire fut acclamé par les élites haïtiennes comme "le nouveau Las Casas et leur seul ami européen ${ }^{89}$.

41 Néanmoins, l'Haïti que Grégoire voulait était vouée à imiter les valeurs européennes (à condition qu'elles soient purifiées). Malgré ses doutes par rapport à la culture européenne, Grégoire ne concevait pas une population non européenne apte à se "guérir par ses propres moyens. Ces peuples nouveaux devraient importer les valeurs européennes et les adapter à leurs propres sociétés, créant ainsi une formule "améliorée de la culture européenne. Grégoire était persuadé que tous les problèmes d'Haïti étaient hérités des colons et qu'après l'effacement de ces corruptions, les Haïtiens seraient comme des "vaisseaux vides prêts à être remplis à nouveau par de nouvelles et meilleures idées venues d'Europe. À ce moment là seulement, les doux et innocents Haïtiens, "les enfants de l'Afrique, pourraient être sur la voie d'un avenir heureux, prospère et stable ${ }^{90}$.

Ses suggestions pour la régénération haïtienne reposaient donc sur les prémisses que les Haïtiens accepteraient le Christianisme et les autres valeurs européennes. Bien qu'il notait lucidement “Je n'ai aucun droit de m'insinuer dans votre gouvernement, l'ancien évêque de Blois ne pouvait se retenir lorsqu'il était question de moralité et de religion ${ }^{91}$. Il opposait les coutumes qualifiées d'arriérées des "peuples idolâtres, musulmans et sauvages, à celles qu'il qualifiait “d'éclairées, venues de l'Europe chrétienne ${ }^{92}$. Il décrivait les Haïtiens comme des êtres naïfs, attachés seulement aux aspects 
superficiels du Christianisme : "on sait que les hommes de couleur et les Noirs aiment la pompe des solennités catholiques et des cérémonies religieuses il leur dit qu'il fallait en observer aussi les préceptes ${ }^{93}$. Tout en se voulant respectueux de la souveraineté haïtienne, il était persuadé faire œuvre salutaire en les encourageant à abandonner la culture de leurs ancêtres. Ainsi, le même universalisme qui lui permettait de considérer les Haïtiens comme ses frères, limitait son aptitude à réaliser l'égalité qu'il affirmait désirer.

La régénération donnait ainsi du pouvoir aux Haïtiens, mais seulement celui de mettre en œuvre les rêves utopiques des Européens éclairés, comme Grégoire. Ainsi, même si Grégoire considérait les Haïtiens comme des êtres humains capables de penser, disposant d'un énorme potentiel, il conforta l'idée selon laquelle ils avaient besoin de la tutelle européenne pour mettre en œuvre ce potentiel. La famille humaine universelle que Grégoire cherchait à construire conduirait finalement les Européens et les autres peuples à la fraternité, mais ces autres peuples devraient abandonner leurs cultures et adopter les valeurs chrétiennes européennes pour faire pleinement partie de l'humanité régénérée. En attendant, ils resteraient les frères cadets de la famille humaine, sujets aux conseils perpétuels de leurs aînés.

Conclusion

44 L'héritage des idées de Grégoire après sa mort reste compliqué. D'un côté, ses arguments en faveur de l'humanité de tous les peuples ont encouragé un nombre considérable de personnes à travers le monde à se battre pour l'abolition de l'esclavage et pour la liberté des peuples contre l'oppression coloniale. Non seulement Grégoire inspira un personnage aussi important que Victor Schœlcher, mais il aida puissamment à galvaniser une nouvelle génération d'abolitionnistes américains ${ }^{94}$. Au xxe siècle, l'influence mondiale de Grégoire ne fut pas limitée à Ho Chi Minh et Aimé Césaire : Jean Price-Mars, le "doyen des intellectuels haïtiens" appelé le "père de la négritude", déclara que les "Haïtiens ont toujours voué un culte fervent à sa mémoire". De la littérature des nègres impressionna tellement l'autodidacte new-yorkais Arturo Schomburg qu'il se consacra à collectionner tous les textes que Grégoire avait évoqués dans cette œuvre. Pareillement, Guichard Parris, un Américain né en Guadeloupe qui commença une thèse de doctorat sur Grégoire dans les années 1930, mais qui finalement devint un dirigeant de la campagne naissante pour les droits civiques aux États-Unis, voyait en Grégoire "the father of Negro literary and biographical history ${ }^{96}$.

Pourtant, malgré la vision positive donnée par Césaire, faisant de Grégoire un des fondateurs de l'anticolonialisme militant, sa conception de la régénération peut être également vue comme donnant aux colonialistes des générations suivantes quelquesuns des outils idéologiques par lesquels ils ont justifié leurs actions. François Manchuelle, qui affirmait que la régénération menait à la fois à la colonisation et au panafricanisme moderne, trouva des liens directs entre la colonisation française moderne en Afrique et les premiers efforts de Grégoire et ses amis pour régénérer ce continent. Dans des opuscules comme la Notice sur la Sierra-Leone de Grégoire, Manchuelle nota que l'abbé avait défendu une sorte de "colonisation philanthropique" qui, à la fois, coloniserait et régénérerait l'Afrique ${ }^{97}$. La régénération exigerait de répandre les bienfaits de la civilisation occidentale, même si les peuples non européens ne savaient pas encore qu'ils en avaient besoin; les exhortations de Grégoire pour annihiler les patois, en France même, et son élévation du français comme l'unique langue d'unité et de culture auraient fourni une nouvelle justification à la colonisation 
française des xixe et xxe siècles. De plus, la régénération forcerait les peuples colonisés à mesurer leurs propres progrès d'après des critères européens. Comme le montre le bas-relief de David d'Angers Les bienfaits de l'imprimerie en Afrique (c. 1840), Grégoire pourrait être imaginé, non pas comme un conseiller à figure de père auprès d'une nation souveraine, mais comme un aventurier colonial portant la civilisation aux peuples infantilisés ${ }^{98}$.

Nous devons donc admettre les composantes paradoxales de la régénération vue par Grégoire, mais également par bon nombre de ses contemporains; elle portait en elle une double dimension et son caractère conflictuel ne doit pas être ignoré dans sa capacité à être simultanément libératrice et contraignante. La régénération permit à Grégoire de mettre en avant l'idée centrale de sa pensée, selon laquelle les peuples de toutes les couleurs avaient la même humanité et des capacités naturelles égales. Mais, en même temps, elle l'amenait à souligner en termes forts les différences existantes. La régénération l'aida à visualiser un monde idéal plein d'harmonie et d'amour, mais elle le conduisit également à considérer comme des ennemis de l'État ceux qui refusaient cet idéal universaliste. La régénération permit à Grégoire d'être simultanément un défenseur courageux de l'autonomie de tous les peuples tout en leur imposant une vision européenne de ce qu'ils devraient aspirer à faire de cette autonomie.

L'importance de la Révolution française dans la genèse de l'universalisme moderne, ne réduit pas ces contradictions à une simple curiosité historique, limitée au personnage de Grégoire ou même à la Révolution. Au contraire, elle continue à avoir des implications aujourd'hui. Le moyen paradoxal par lequel la régénération - et la Révolution elle-même - traita de la différence ne fut jamais complètement résolu. Même si nous créons des sociétés ouvertes à tous, nous n'avons pas encore trouvé comment réconcilier les différences humaines avec des systèmes affirmant l'égalité. C'est une des principales raisons pour lesquelles Grégoire continue à attirer l'attention $\mathrm{du}$ monde moderne, et c'est pourquoi son héritage restera le lieu de contestations passionnées.

\section{NOTES}

1.Cet article propose une synthèse de quelques-uns des thèmes que j'ai développés dans ma thèse de doctorat, "Regenerating France, Regenerating the World : the abbé Grégoire and the french Revolution, 1750-1831", Stanford University, Department of History, 1998. Je remercie ici tous les participants au congrès international sur l'abbé Grégoire, qui s'est tenu à la Clark Library à Los Angeles en février 1997, pour leurs réponses stimulantes aux idées exprimées dans cet article. Je suis particulièrement reconnaissante à Marcel Dorigny de m'avoir encouragée à préparer cet article et pour son aide à la préparation d'une version française. Ma gratitude va également à Keith Michael Baker, David A. Bell, Dena Goodman, Jennifer Heuer, Mary Louise Roberts, Aron Rodrigue, Peter Sahlins et Joseph Zizek pour leurs précieuses suggestions sur des versions préliminaires de ce texte. 
2.Bernard PlOngeron, L'abbé Grégoire ou l'Arche de la fraternité, Paris, 1989, p.22.

3.Annie KRIEGEL, “Un hommage critiquable, Le Figaro, 12 décembre 1989, p.2 ; Samuel TRIGANo, “The French Revolution and the Jews, Modern Judaism, 10, 1990, pp.171-190.

4.J'emprunte ici la notion des paradoxes d'universalisme à Joan Wallach ScotT (Only Paradoxes to Offer : French Feminists and the Rights of Man, Cambridge, MA, 1996).

5.Cet article ne tente pas de donner une narration biographique détaillée sur Grégoire. Voir, parmi d'autres, Louis MAGGIOLO, “L'abbé Grégoire, 1750-1789, Mémoires de l'Académie de Stanislas, 1873, 1883, et 1884 ; Ruth NECHELES, The abbé Grégoire 1787-1831. The Odyssey of an Egalitarian, Westport, CT, 1971 ; et Maurice EzRAN, L'abbé Grégoire, défenseur des juifs et des Noirs : révolution et tolérance, Paris, 1992.

6.J'ai développé plus longuement cette partie de la vie de Grégoire dans ma thèse.

7.Mémoires de l'abbé Grégoire, éd. J. M. Leniaud et préface par J. N. Jeanneney, Paris, 1989, pp.117-118.

8.Sur l'éducation de Grégoire, voir Louis MAGGIOLO, op. cit. (1873) ; et Antoine SUTTER, Les années de jeunesse de l'abbé Grégoire : son itinéraire jusqu'au début de la Révolution, Sarreguemines, 1992.

9.Voir ses Observations sur le décret de l'Assemblée nationale qui ordonne une nouvelle circonscription des Paroisses, Paris, 1790, 14.

10.Sur ce concours célèbre, voir l'article classique d'Abraham CAHEN, "L'émancipation des juifs devant la société royale des sciences et des arts de Metz en 1787 et M. Roederer, Revue des études juives, I, 1880, pp.83-104.

11.Sur "régénération" comme baptême, voir Dictionnaire universel d'Antoine Furetière [réimpression de l'édition de 1690], Paris, 1978, t. III et sa version révisée du XVIIIe siècle, Dictionnaire universel françois et latin, vulgairement appellé Dictionnaire de Trévoux...nouvelle édition corrigée et considérablement augmentée, Paris, 1743, V, 858. Sur "régénération" comme baptême mais aussi résurrection, voir M.l'abbé BERGIER, Encyclopédie Méthodique. Théologie, Paris, 1790, III, 335. Sur les définitions médicales, voir Dictionnaire historique de la langue française, Paris, 1992, II, 1747 et Grand Larousse de la langue française, Paris, 1977, VI, 4993. Pour les usages des philosophes et historiens naturels, $c f$. Jean Le Rond D'ALEMBERT, Discours préliminaire de l'Encyclopédie, Paris, 1751, XIX-XX, et Charles BONNET, La palingénésie philosophique, ou idées sur l'état passé et sur l'idée future des êtres vivants, ouvrage destiné à servir de supplément aux derniers écrits de l'Auteur, et qui contient principalement le précis de ses recherches sur le christianisme, Genève, 1769. 12.Voir Antoine DE BAECQUE, Le Corps de l'histoire : métaphores et politique (1770-1800), Paris, 1993, p.171 ; et Mona Ozouf, "Régénération, dans Dictionnaire critique de la Révolution française, éd.François Furet et Mona Ozouf, Paris, 1988.

13.Dans ses écrits sur Grégoire (par exemple "Préface, dans l'édition de Flammarion de l'Essai sur la régénération physique, morale et politique des juifs, Paris, 1988), Rita HERMONBELOT a soutenu que les influences principales sur la pensée de Grégoire au sujet des juifs étaient toujours jansénistes, mais il y a plus d'évidence à soutenir cette position pour la période post-révolutionnaire que pour la période antérieure. Sur la "conversion de Grégoire au jansénisme après Thermidor, voir aussi Catherine MAIRE, De la cause de Dieu à la cause de la Nation : le jansénisme au XVIIIe siècle, Paris, 1998, pp.586-587 et passim. 14.Essai sur la régénération (édition Hermon-Belot), p.117.

15.Ibid, p.94.

16.Ibid, p.177.

17.Ibid, p.131. 
18. Voir Ibid, p.195 et John ToLAND, Reasons for Naturalizing the Jews in Great Britain and Ireland, On the same foot with all other Nations. Containing also, a Defence of the Jews against All vulgar Prejudices in all Countries, London, 1714 ; réimpression Jérusalem, 1963, pp. 45-46 et passim. Parmi les autres ouvrages classiques sur les juifs qui ont influencé Grégoire, on peut compter ceux de Jacques Basnage, Pierre-Louis Lacretelle, et Christian Wilhelm Dohm.

19.Toland avait suggéré que la moralité des juifs était peut-être meilleure que celle du sujet britannique moyen, ibid, pp.10-11, 52.

20.Essai sur la régénération, pp.58, 61.

21.Ibid, p.161. Voir la discussion de HERMON-BELOT sur la présence de juifs posant "un réel problème intellectuel pour Grégoire et les autres chrétiens : “L'abbé Grégoire et la conversion des juifs, dans Les juifs et la Révolution française : histoire et mentalités, éd. Evelyne Oliel-Grausz et Mireille Hadas-Lebel, Louvain, 1992, p.22.

22.Essai sur la régénération, p.75.

23.D'après les règles kaschers, les juifs ne sont autorisés à manger aucune trace de sang, et doivent saler les viandes avant de les manger afin d'enlever tout le sang.

24. Essai sur la régénération, p.72.

25.Ibid, p.83.

26.Ibid, p.85.

27.Ibid, p.96.

28.Ibid, p.138. Grégoire pensait, pourtant, que si les chrétiens autour d'eux étaient immoraux, les juifs suivraient leur exemple.

29.Ibid, p.134.

30.Ibid, p.149.

31.Timothy TACKETT, Becoming a Revolutionary : The deputies of the French National Assembly and the Emergence of a Revolutionary Culture (1789-1790), Princeton, 1996, pp.23 et 286.

32. Sur cette synthèse, voir B. PLONGERON, op. cit.

33.Discours prononcé le jour de Toussaint 1789, en l'église de l'Abbaye de Saint-Germain-desPrés, pour la bénédiction des quatre flammes de la milice nationale de ce district, Paris, 1789, p. 5.

34.Grégoire a publié une Motion en faveur des juifs (Paris, 1789) et a essayé de soulever le sujet de la situation juive dans l'Assemblée à l'automne de 1789, sans succès.

Néanmoins, pendant les trois moments où l'Assemblée entière a discuté de donner ou non la citoyenneté aux juifs (déc.1789, janv. 1790 et sept. 1791), Grégoire ne dit presque rien. Il est probable qu'il fût empêché de parler en décembre 1789, mais plus tard lors des autres débats il semble simplement qu'il n'a pas voulu risquer ses plus grands projets en luttant pour une cause notoirement impopulaire. Voir Courrier de Paris dans les provinces du 28décembre 1789, pp. 254-256 ; et NECHELES, op. cit., pp.27-33.

35.Voir par exemple son Dessèchement des marais, défrichements et plantations. Observations sur le Rapport du Comité d'Agriculture, s.l., 1790 ; et A.P., tome XII, 30 mars 1790, p. 458.

36. Essai sur la régénération, p.154.

37.Enquête sur les patois, Archives départementales de Loir-et-Cher [“ADLC”], F 1273, fol. 5 Voir également Le Patriote français, 23 août 1790, p. 2.

38. Moniteur, 11 février 1790, 166-7. Voir aussi Michel DE CERTEAU, Dominique JULIA, et Jacques REVEL, Une politique de la langue. La Révolution française et les patois : l'enquête de Grégoire, Paris, 1975 ; et David A. BELL, “Lingua Populi, Lingua Dei : Language, Religion 
and the Origins of French Revolutionary Nationalism, American Historical Review, 100, 1995, pp.1403-1437.

39. Motion en faveur des juifs, 43.

40.A.P., tome XII, 28 mars 90, p.383.

41. Mémoire en faveur des gens de couleur ou sang-mêlés de Saint-Domingue et des autres Isles françaises de l'Amérique, adressé à l'Assemblée nationale, Paris, 1789, p.8.

42.Ibid., pp.38-39.

43. Notice sur la Sierra-Leone et sur une calomnie répandue à son sujet contre le gouvernement français. Extrait de la décade philosophique, s.1., 1796, p.5.

44.A.P., tome XXV (11 mai 1791), p. 740. Même s'il y avait beaucoup d'esclaves mulâtres et un assez large nombre des Noirs libres, Grégoire souvent réduisait "esclave" à "nègre," et "libre" à "mulâtre".

45. Mémoire en faveur des gens de couleur, p.38.

46.Lettre aux Philanthropes, Paris, 1790, p.4.

47. Lettre aux citoyens de couleur et nègres libres de Saint-Domingue, et des autres isles françaises de l'Amérique, Paris, 1791, pp.12-13. En décembre 1789, Grégoire se trouvait en désaccord avec d'autres membres de la Société des Amis des Noirs, en préconisant qu'une campagne pour obtenir l'interdiction du commerce des esclaves soit entreprise aussitôt que "l'affaire des citoyens de couleur serait terminée" (voir Marcel DoRIGNY, “L'abbé Grégoire et la Société des Amis des Noirs d'après les archives de la Société, communication au colloque sur l'Abbé Grégoire, Clark Library, Los Angeles, février 1997. Voir également Marcel DoRIGNY et Bernard GAINOT, La Société des Amis des Noirs. 1788-1799. Contribution à l'histoire de l'abolition de l'esclavage. Paris, 1998, édition Unesco. 48.Abraham FURTADO et al., Lettre adressée à M. Grégoire... par les députés de la Nation juive portugaise de Bordeaux, Versailles, 1789, p.3.

49.Lettre au Comité de constitution sur l'affaire des juifs, par M. de Bourge, représentant de la commune de Paris, Paris, 1790, p. 7.

50.Grégoire avait plus d'espoir que beaucoup d'autres jacobins dans la nouvelle Constitution, bien qu'il ait éprouvé du ressentiment que le Roi y conservait une grande partie de son pouvoir. Voir ses remarques dans A.P., tome XXVIII (15 juillet 1791), pp. 318-320 et tome XXIX (12 août 1791), pp.383-384.

51.Pour plus des détails sur le Christianisme révolutionnaire de Grégoire, voir B. PLONGERON, op. cit.

52.M. Ozouf, “La Révolution française et la formation de l'homme nouveau, dans L’homme régénéré. Essais sur la Révolution française, Paris, 1989, pp.116-157.

53.A.P., tome XXII (4 janvier 1791), p. 14.

54.Moniteur, 27 décembre 1790, p.1493.

55.Lettre pastorale de M. l'évêque du département de Loir-et-Cher, 24 mars 1791, Blois, 1791, p. 6.

56.Discours sur la fédération du 14 juillet 1792, Orléans, [1792], p.5.

57. “Aux bons citoyens du département de la Meurthe, 6 août 1790, Le Patriote français, 11 août 1790, p.4.

58.Adresse aux habitants du Valais, Chambéry, [1793], p.5.

59.Discours prononcé dans l'église cathédrale de Blois... au service célébré pour JacquesGuillaume Simonneau, maire d'Étampes, assassiné le 3 mars 1792, pour avoir défendu la loi, Blois, 1792, pp.19-20.

60.A.P., tome LII, 21 septembre 1792, p. 73. 
61. Voir les réponses de Grégoire aux délégations britanniques et allemandes en novembre 1792 (A.P., tome LIII) comme celles des 7 nov. (275); 10 nov. (341); 15 nov. (418) ; et 21 nov. (635-7). Grégoire était président de la Convention pendant la dernière moitié de ce mois.

62. Adresse de l'assemblée administrative du département de Loir-et-Cher, réunie en Conseil général, à ses concitoyens, le 1er août 1792, Blois, 1792, conservée aux ADLC, F 1275. La brochure a été composée suivant une motion de Grégoire à accroître le nombre de volontaires (voir ADLC, L 109, 24 juillet 1792).

63.Grégoire et Jagot au maire de la commune de Menton, le 4 avril 1793, dans Henri MoRIs, Organisation du département des Alpes-Maritimes formé du ci-devant comté de Nice et de la ci-devant principauté de Monaco. Mars - avril 1793. Lettres des représentants du peuple Grégoire et Jagot, chargés de cette organisation, Paris, 1915, p.39. Moris a trouvé ces lettres aux AN, D\$1 25, dossier 1, et a affirmé que Grégoire "semble être l'auteur de tout le recueil(XXIV).

64.Grégoire et Jagot à la société populaire de Menton, le 18 avril 1793, ibid, p.61.

65.Grégoire et Jagot au citoyen Bizanet, commandant militaire à Monaco, le 12 avril 1793, ibid., p.50.

66. Voir M. J. Guillaume, Procès-verbaux du Comité d'instruction public de la Convention nationale, Paris, 1891 - 1907, t. III, p.41 (9 frimaire an II).

67.Comme François Souchal l'a remarqué, "Grégoire vint, un peu sur le tard, mais il sut si bien organiser sa publicité et façonner son image pour les générations futures qu'on lui attribua assez généralement la paternité d'une lutte généreuse et intrépide contre le vandalisme..., Le vandalisme de la Révolution, Paris, 1993, p.115.

68.Sur Grégoire et l'Église constitutionnelle, voir B. PLONGERON, op. cit. Augustin GAZIER, Études sur l'histoire religieuse de la Révolution française d'après des documents originaux et inédits, Paris, 1887 et Virginie MunduTEGUY, "Recherche sur l'Église constitutionnelle post-thermidorienne à travers une société et un périodique [An III - An VI], mémoire de maîtrise, Institut d'histoire de la Révolution française, Université de Paris-I, 1996, sous la direction de Catherine Duprat.

69.Voir parmi d'autres ouvrages ses Essais historiques sur les libertés de l'église gallicane, Paris, 1818, et Histoire des confesseurs des empereurs, des rois, et d'autres princes, Paris, 1824. 70.M. LASCARIS, "L'abbé Grégoire et la Grèce, La Révolution française, 85, 1932, pp. 220-231. 71.Voir par exemple sa correspondance avec Guilian Verplanck, New York Historical Society, Guilian Verplanck Papers; et avec la Pennsylvania Society for the Abolition of Slavery, Historical Society of Pennsylvania, Pennsylvania Abolition Society Papers, AMS081. Voir aussi Thomas Jefferson à Grégoire, 25 février 1809, publiée dans Paul FORD, The Writings of Thomas Jefferson, New York, 1898, IX, p.246.

72.Voir par exemple "Articles recommandés à la bienveillance de M. Michaux", Benjamin Vaughan Papers, 46 p., American Philosophical Society, Philadelphia ; et la correspondance de Grégoire avec l'archevêque John Carroll, archevêché de Baltimore, introduite et publiée par Jacques M.GRES-GAYER, "Four Letters from Henri Grégoire to John Carroll, 1809-1814", Catholic Historical Review 79, n 4, 1993, pp. 681-703.

73.PLONGERON, op. cit., pp.34-35.

74.Aimé CÉSAIRE, "Discours d'inauguration de la place de l'abbé Grégoire. Fort-deFrance - 28décembre 1950, Euvres complètes, Paris, 1976, pp.422-423. Je suis reconnaissante au professeur Hans-Jürgen Lüsebrink pour m'avoir signalé ce discours. 
75.Voir la discussion de B. PLONGERON sur la "passion pour la réunion" et rassemblement dans l'Église de Grégoire, op. cit., surtout "Une Pentecôte des Nations pour la République, pp.37-50.

76.Mémoires, p.53

77.Observations nouvelles sur les juifs, et spécialement sur ceux d'Allemagne, s.l., 1806, p.7 ; Observations nouvelles sur les juifs, et spécialement sur ceux d'Amsterdam et de Francfort, s. 1., 1807, p.4.

78.Saltzmann à Grégoire, 21 mars 1806, BSPR-G, dossier "Bas-Rhin."

79.Grégoire à Hannah Adams, septembre 1810, extraite dans ADAMS, The History of the Jews from the Destruction of Jerusalem to the Nineteenth Century, Boston, 1812, II, p. 152 [Adams ne fournissait pas l'original français de la lettre de Grégoire, seulement une traduction anglaise].

80.Grégoire à Joël Barlow, 1er septembre 1806, Houghton Library, Harvard University, JoëlBarlow Papers, bMS AM 1448, fol. 606, citée avec permission de la Houghton Library.

81. De la liberté de conscience et de culte à Haïti, Paris, 1824, p.30.

82.Frances MALINo, A Jew in the French Revolution : The Life of Zalkind Hourwitz, Oxford, 1996, pp.52 et passim.

83.Berr-Isaac BERR, Lettre du sieur Berr-Isaac-Berr, manufacturier, membre du conseil municipal de Nancy, à M. Grégoire, sénateur, à Paris, Nancy, 1806, pp.5, 6 et 9. Sur Berr, voir aussi Martine LEMALET, "Berr Isaac Berr : Un bilan contemporain de l'émancipation, dans Les juifs et la Révolution française : histoire et mentalités, pp.119-138.

84.Sur l'enthousiasme des juifs français pour l'idée de régénération au xıxe siècle, voir Jay R.BERKOVITZ, The Shaping of Jewish Identity in Nineteenth-century France, Détroit, 1989 ; et Aron RodRIGUE, French Jews, Turkish Jews, Bloomington, IN, 1990.

85.La plus grande partie de cette correspondance est conservée à la Bibliothèque de l'Arsenal, Mss. 6339 et 15049.

86.Arsenal, Ms. 15049, fol. 185, 189.

87.Voir Grégoire à une personne non identifiée, 15 avril 1815, B.N.F., nouvelles acquisitions françaises, ms. 24910, fol. 288.

88.De la liberté de conscience, p.42.

89. Comte de Limonade à Grégoire, 10 juin 1814 ; et Inginac à Grégoire, 1er avril 1820, Arsenal, Ms.6339, fol. 44, 81.

90.Hans-Jürgen LÜSEBRINK a fait un raisonnement similaire sur l'anticolonialisme et paternalisme simultanés chez Grégoire dans son article, "Negrophilie und Paternalismus : die Beziehungen Henri Grégoire zu Haiti (1790-1831), dans Der Karibische Raum zwischen Selbst- und Fremdbestimmung : Zur Karibischen Litteratur, Kultur und Gesellschaft, éd. Reinhard Sander, Frankfurt am Main, 1984, pp.99-108. 91.Grégoire au président Jean-Pierre Boyer, 22 juin 1821, Arsenal, Ms. 15049 / 169. La propension de Grégoire à donner des conseils était bien différente de son allié abolitionniste Lafayette, qui a correspondu avec le président d'Haïti comme un général à un autre, sans donner ses avis sur les affaires intérieures haïtiennes. Voir la correspondance Lafayette-Boyer, Rare Book and Manuscript Collections, Cornell University Library, Ithaca, New York, 4611 Boxes 14, 25 et 27.

92.De l'influence du christianisme sur la condition des femmes, 3e éd. Paris, 1829, pp.131 et passim.

93. Considérations sur le mariage et le divorce adressées aux citoyens d'Haïti, Paris, 1823, pp. $42-43$. 
94.Voir "Introduction", On the Cultural Achievements of Negroes, traduction de De la littérature des nègres, éd. Thomas Cassirer et Jean-François Briere, Amherst, MA, 1996, XV - XLVIII.

95.Dr. Jean PRICE-MARS, Silhouettes de Nègres et de Négrophiles, Paris, 1943, p.198.

96.Parris à Duraciné Vaval, 12 janvier 1937, New York Public Library, Schomburg Center for Research in Black Culture, Parris Collection, Box 1, dossier "Grégoire Correspondence".

97.François MANCHUELLE, “The Regeneration of Africa : An Important and Ambiguous Concept in 18th and 19th Century French Thinking about Africa", Cahiers d'études africaines 144, $\mathrm{n}^{\circ}$ XXXVI-4, 1996, pp. 559-588. Sur les projets de colonisation civilisatrice de Grégoire et des autres abolitionnistes, voir aussi Yves BÉNOT, La démence coloniale sous Napoléon, Paris, 1991. Voir également, Marcel DoRIGNY, "La Société des amis des Noirs et les projets de colonisations en Afrique, Annales historiques de la Révolution française, septembre-décembre 1993.

98.Un moulage de ce bas-relief est placé à la base de la statue de la place Gutenberg à Strasbourg; on peut également voir ce bas-relief au musée David d'Angers, à Angers. Voir également dans Jean METELLUS et M. DORIGNY, De l'esclavage aux abolitions. XVIIIe-XXe siècle, Paris, 1998, éditions Cercle d'art : le bas-relief de David d'Angers est reproduit à la page 138.

\section{RÉSUMÉS}

Cet article propose une nouvelle perspective sur l'abbé Grégoire et sur les débats qui ont entouré sa panthéonisation en 1989, en analysant son idée de régénération. L'auteur examine l'idée même de régénération dans l'œuvre de Grégoire, de ses racines prérévolutionnaires à la Révolution, et analyse ses conceptions régénératrices en direction de groupes opprimés en France (comme les juifs, les Noirs, les habitants de pays à idiomes) mais également à l'égard de la Nation française et du monde entier. L'auteur met en particulier en relief les paradoxes inhérents à la notion de régénération - sa faculté à la fois libératrice et contraignante - et conclut en soulignant que ces contradictions sont encore aujourd'hui en France au cœur des actions prises en faveur de la "différence culturelle.

The paradoxes of the revolutionary regeneration : the abbé Grégoire case.

This article gives a new approach to the abbé Grégoire case and to the debates concerning his pantheonization in 1989 through an analysis of his idea of regeneration. The author studies thevery idea of regeneration in Gregoire's work, from its pre-revolutionary origins to the French Revolution and analyses his regenerating conceptions towards oppressed people in France (such as Jews, Blacks, dialect speaking inhabitants), the French Nation and the world as well. The author emphasizes the paradoxes linked with the idea of regeneration as it is both liberating and constraining. She concludes by stressing the fact that today in France these contradictions are still parts of the actions in favor of the cultural differences. 
INDEX

Mots-clés : abbé Grégoire, gens de couleurs, Haïti, juifs, régénération, religion

AUTEUR

ALYSSA GOLDSTEIN SEPINWALL

California State University 